\title{
Experimental Study of a Sphere Bouncing on the Water
}

\author{
Xujian Lyu ${ }^{1} \cdot$ Honglu Yun ${ }^{1,2} \cdot$ Zhaoyu Wei $^{2}$
}

Received: 15 March 2021 / Accepted: 6 August 2021 / Published online: 1 January 2022

(c) The Author(s) 2021

\begin{abstract}
In this paper, the flow physics and impact dynamics of a sphere bouncing on a water surface are studied experimentally. During the experiments, high-speed camera photography techniques are used to capture the cavity and free surface evolution when the sphere impacts and skips on the water surface. The influences of the impact velocity $\left(v_{1}\right)$ and impact angle $\left(\theta_{1}\right)$ of the sphere on the bouncing flow physics are also investigated, including the cavitation evolution, motion characteristics, and bounding law. Regulations for the relationship between $v_{1}$ and $\theta_{1}$ to judge whether the sphere can bounce on the water surface are presented and analyzed by summarizing a large amount of experimental data. In addition, the effect of $\theta_{1}$ on the energy loss of the sphere is also analyzed and discussed. The experiment results show that there is a fitted curve of $v_{1}=17.5 \theta_{1}-45.5$ determining the relationship between the critical initial velocity and angle whether the sphere bounces on the water surface.
\end{abstract}

Keywords Water entry $\cdot$ Bouncing sphere $\cdot$ Critical impact angle $\cdot$ High-speed photograph $\cdot$ Splash

\section{Introduction}

The impact dynamics of structures on the water have been studied for several decades because of their wide engineering applications (Glasheen and Mcmahon 1996; Miloh and Shukron 1991; Shlien 1994; Von Karman 1929), such as the landing of a seaplane and a speedboat gliding on water. Bouncing and skipping a stone and the swift running of insects or basilisk lizards are other interesting natural phenomena on a water surface that attract the attention of scientists (Clanet et al. 2004, Glasheen and Mcmahon 1996). The bouncing mechanism of an elastic sphere on a

\section{Article Highlights}

- In order for the spheres entering into water with stability, the phenomenon of bouncing should be avoided.

- High-speed photography and methods of force analysis based on these images are deployed to reveal the performance of spheres bouncing on the water surface.

- There is a critical curve indicating the relationship between the initial velocity and angle to determine whether the sphere bounces on the water surface.

$\triangle$ Zhaoyu Wei

wzhyu@sjtu.edu.cn

1 School of Energy and Power Engineering, Nanjing University of Science and Technology, Nanjing 210094, China

2 School of Oceanography, Shanghai Jiao Tong University, Shanghai 200240, China water surface was studied by Belden et al. (2016) through experiments and numerical simulations. They found that the bouncing dynamics is decided by the ratio of the material shear modulus to hydrodynamic pressure and the wave propagation speed. Hurd et al. (2019) revealed a new mode of bouncing on the water for relatively soft spheres with low impact angles by experiments, which shows good agreement between measured acceleration, number of skipping events, and distanced traveled. Liu and Smith (2014) proposed a new prediction formula for repeated impacts and rebounds, especially over short time scales. Korobkin et al. (2011) summarized and analyzed the mathematical problems of modern hydroelastic mechanics and analyzed some models and techniques. Hewitt et al. (2011) experimentally studied the planning and bouncing of rectangular paddles on a water surface and established a model using shallow water theory. By studying the rebound effect of objects in the shallow layer of a liquid, Hicks and Smith (2011) proposed a model that couples the motion of an object to fluid dynamics.

In addition, projectile and stone skipping is also quite an interesting phenomenon that attracts the attention of researchers. Nishida et al. (2010) studied the dynamic properties of a projectile impinging on a granular medium and derived a formula for determining the critical angle by the density ratio and diameter ratio through experimental techniques. Rosellini et al. (2005) studied the hydrodynamic characteristics of a rotating disk impacting water with 
multiple bounces. They found that the source of dissipation lies in the dependence of this reaction force on the angle between the water surface and the trajectory of the stone. In addition, they proposed a simple model to measure bounce dissipation. Bocquet (2003) analyzed the physics of stone skipping and found that the maximum number of bounces can be estimated by the deceleration and angular stability of the stone. Faltinsen (2000) reviewed hydroelastic slamming experiments and theoretical studies and noted that hydrodynamics research on slamming problems must be from a structural point of view. Johnson (1998) summarized the theory of ricochet from a liquid surface of a non-rotating sphere and improved the non-rotating projectile portion. Truscott et al. (2014) summarized experimental, theoretical, and numerical studies of an object impacting on the water and introduced several problems to be solved, including the high-fidelity measurements of acceleration, surface stress, and cavity pressure.

The above literature shows that most of the studies addressed aspects of the law of bouncing plate-shaped objects on the water, for instance, the number of bounces. The phenomenon of cavity evolution and kinetic energy dissipation of a symmetric and regularly shaped object (i.e., a sphere) is further from being clearly explained. This forward-looking paper reveals the cavity dynamics and the mechanism of a sphere bouncing on a water surface. Experimental evaluation of the effects of the initial impact angle and the energy dissipation and estimation of the law of the critical impact angle are provided for the bouncing of a sphere on a free water surface.

\section{Experimental Methods of High-Speed Photograph}

The experiment was conducted in a water tank located in Nanjing University of Science and Technology, China. During the experiments, the temperature was 23 to $27{ }^{\circ} \mathrm{C}$, and the closed laboratory eliminated the interference of natural light and airflow. The size of the tank was $1500(L) \times$ $400(W) \times 600 \mathrm{~mm}(H)$, and the water depth was set to $350 \mathrm{~mm}$, as shown in Figure 1. The water tank was made of $10-\mathrm{mm}$ thick optical glass to reduce the optical path loss. The steel sphere used in the experiment has a mass $m \approx 1.397 \mathrm{~g}$, a diameter $d \approx 7 \mathrm{~mm}$, and density $\rho_{s} \approx 7778.6$ $\mathrm{kg} / \mathrm{m}^{3}$. The measurement error of these parameters was less than $0.5 \%$. The sphere was launched by an electromagnetic launcher similar to the equipment described in Yun et al. (2020), and the arrangement is shown in Figure 1(a).

A high-speed digital video camera, Phantom VEO410L, with a $105-\mathrm{mm}$ fixed-focus Nikon lens and a resolution of $1280 \times 400$ pixels, was used to capture the sphere's movement and the associated cavity evolution. The horizontal distance

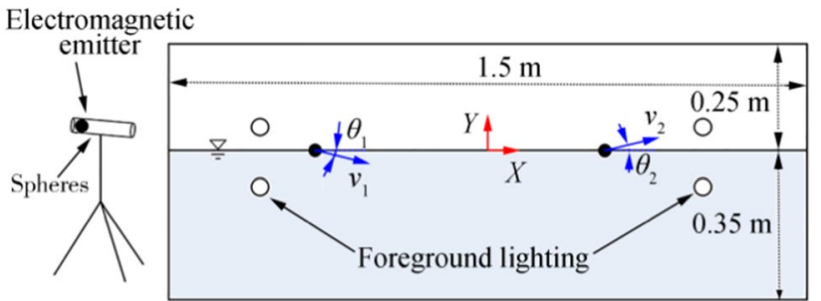

(a) Front view

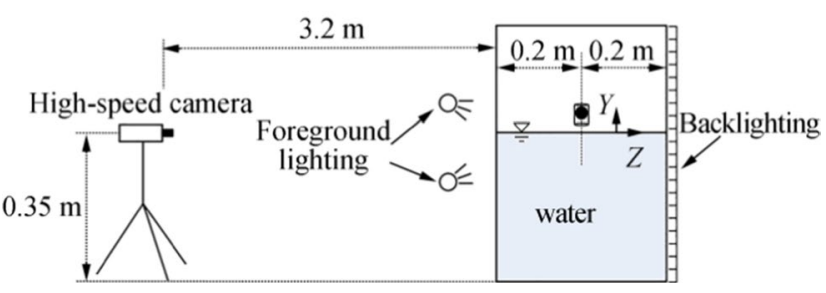

(b) Size view

Figure 1 Sketch of the experimental setup

between the camera and the electromagnetic launcher was $3.4 \mathrm{~m}$, which allows the microlens to capture the whole flow physics during the impact process, and the camera center was approximately $0.35 \mathrm{~m}$ above the ground, which is nearly the same height as the free water surface. For the quality of the flow visualization experiment, an adequate supplementary light source is fundamental. In the experiment, four highfrequency lamps of the type JINBEI EF-200 LED and a rectangular LED light box were used to provide sufficient light intensity. Four high-frequency lamps were used as the foreground lighting arranged in front of the water tank, and the light box was used as the backlighting to provide a uniform white background arranged at the back of the water tank, as shown in Figure 1. On the basis of these strong lightings, the frame rate was set as high as 10000 fps to capture significantly crisp images to accurately analyze the trajectory of spheres, and the exposure time was decreased to $10 \mu \mathrm{s}$.

Seven typical cases with similar initial impact velocities and different impact angles were selected as analysis objects, and the parameters are shown in Table 1 . The main parameters include the initial impact velocity $v_{1}$, initial impact angle $\theta_{1}$, bouncing velocity $v_{2}$, and bouncing angle $\theta_{2}$; definitions of the variables (i.e., $v_{1}, \theta_{1}, v_{2}$, and $\theta_{2}$ ) are shown in Figure 1(a).

\section{Results and Discussion on the Sphere Bouncing and Cavity Characteristics}

Cavity dynamic characteristics and the kinematics law of a steel sphere bouncing on the water will be discussed in this section. 
Table 1 Main parameters of typical cases

\begin{tabular}{llccc}
\hline Case list & $v_{1}(\mathrm{~m} / \mathrm{s})$ & $\theta_{1}\left(^{\circ}\right)$ & $v_{2}(\mathrm{~m} / \mathrm{s})$ & $\theta_{2}\left(^{\circ}\right)$ \\
\hline 1 & 58.58 & 1.04 & 56.81 & 0.94 \\
2 & 58.41 & 2.60 & 50.86 & 2.68 \\
3 & 58.66 & 3.69 & 47.42 & 3.26 \\
4 & 56.69 & 4.53 & 38.75 & 4.32 \\
5 & 57.88 & 5.89 & 6.22 & 5.57 \\
6 & 57.68 & 6.01 & - & - \\
7 & 56.78 & 10.23 & - & - \\
\hline
\end{tabular}

\subsection{Cavity Formation of a Sphere Bouncing on the Water}

To analyze the hydrodynamic characteristics of the sphere, $t_{0}$ is used to indicate the time when the sphere just touches the water surface. Figure 2(a) shows the selected image sequence for case 1 with an initial impact velocity of $v_{1}=58.58 \mathrm{~m} / \mathrm{s}$ and an initial impact angle of $\theta_{1}=1.04^{\circ}$. Because of the very small initial impact angle and the larger initial impact velocity, the center of the sphere was always above the static-free surface throughout the bouncing process, and the sphere was in contact with water for a short period of only $1.5 \mathrm{~ms}$. The cavity is seen to be the shallowest and the splash is weak. Furthermore, the bouncing velocity of $v_{2}=56.81 \mathrm{~m} / \mathrm{s}$ is quite near the initial impact velocity of $v_{1}=58.58 \mathrm{~m} / \mathrm{s}$. The bouncing angle of $\theta_{2}=0.94^{\circ}$ is near the impact angle, indicating that a slight amount of energy is lost during the water surface impact.

The typical images of case 2 are shown in Figure 2(b), and the initial impact angle of the sphere was $2.60^{\circ}$. It can be shown that the splash above the free surface is much stronger, and the cavity is relatively deep and large compared
Figure 2 Cavity evolution of a sphere bouncing on the water for respective cases 1 to 4

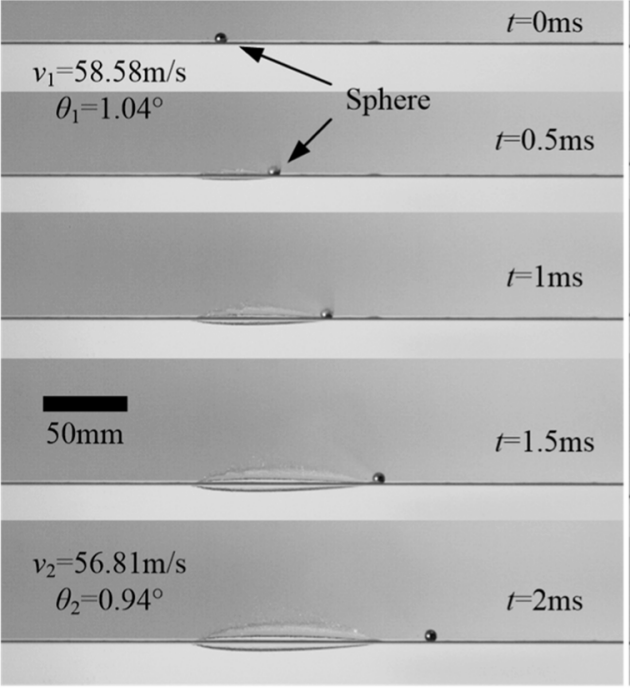

(a) Case 1

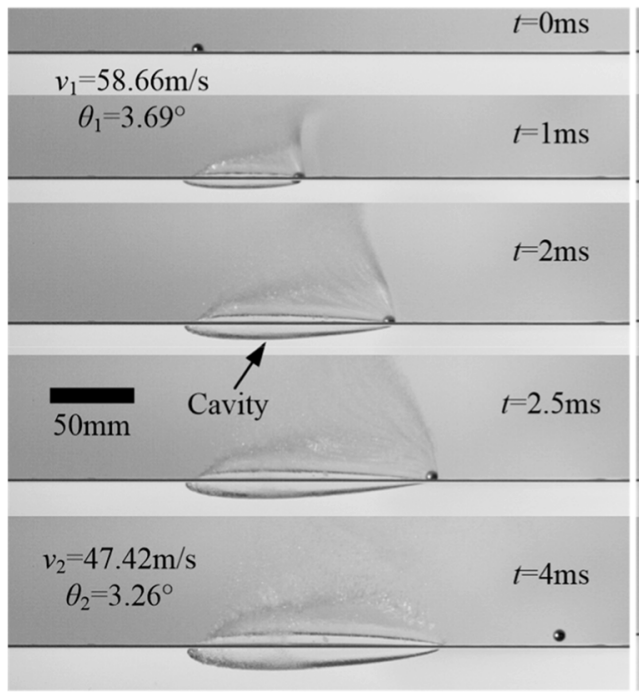

(c) Case 3

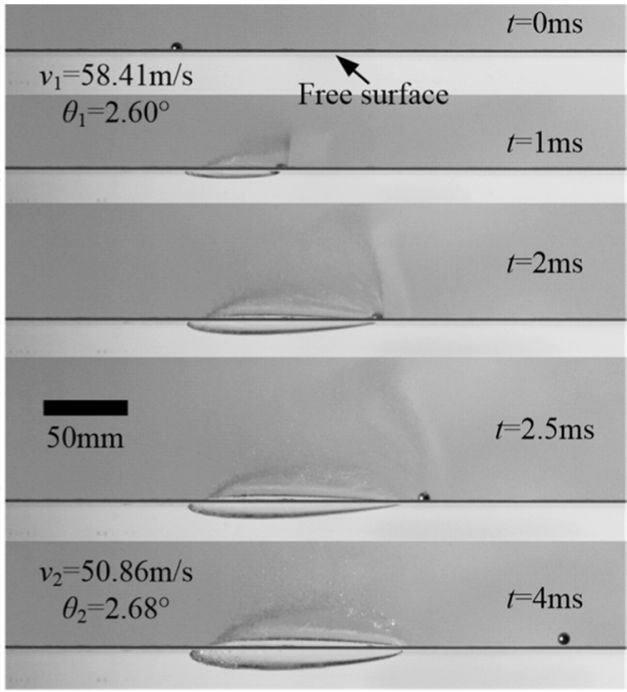

(b) Case 2

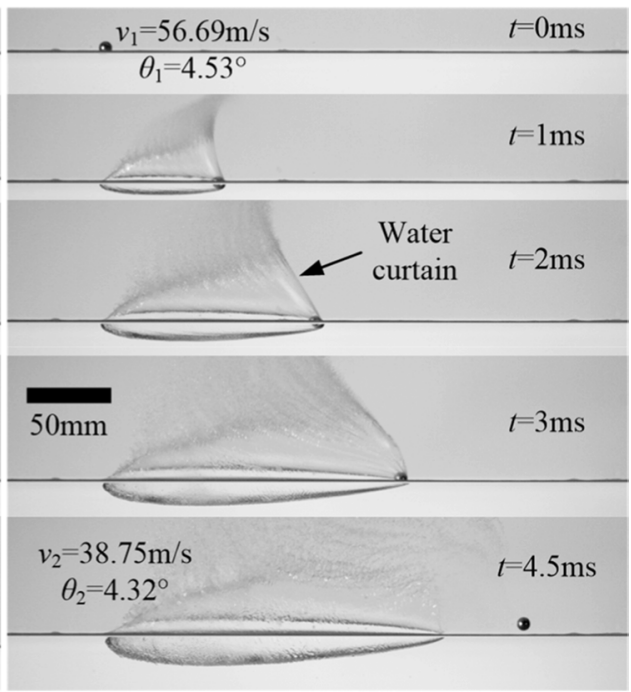

(d) Case 4 
with that of case 1 . Moreover, the duration of the sphere touching the water is extended slightly to $\Delta t=2.5 \mathrm{~ms}$, compared with $\Delta t=1.5 \mathrm{~ms}$ for case 1. Figure 2(c) and (d) show the selected image sequences for case 3 and 4 , respectively. Note that the initial impact velocities for the four cases are similar and the initial impact angles are gradually increased. It is seen that the larger the initial impact angle is, the deeper and larger the profile of the cavity formed. Furthermore, it can be seen in case 4 that a smooth and complete water curtain is formed, which almost encloses the entire sphere as it leaves the water surface (Figure 2(d)). Another significant phenomenon seen in cases 1 to 4 is that the bouncing velocity decreases as the initial impact angle increases, although the initial impact velocity is the same in each case.

It is obvious that the flow physics of case 5 is different from those of the other cases, as shown in Figure 3. For case $5, v_{1}$ is $57.88 \mathrm{~m} / \mathrm{s}$ and $\theta_{1}$ is $5.89^{\circ}$. Notably, the duration that the sphere moved under the free surface is much longer for case 5, reaching $46 \mathrm{~ms}$. Furthermore, the sphere is displaced $675 \mathrm{~nm}$ in the water, nearly 100 -fold its diameter. A complete cavity, including the smooth water film above the static water surface, encloses the sphere as it moves in the water. The cavity wall behind the sphere finally becomes rough because of the cavity collapse and the effect of capillary waves on the free surface, as described by Grumstrup et al. (2007).

The cavity finally collapses to many small air bubbles, which is possibly associated with the hydrostatic pressure under the free surface. The cavity also causes violent-free surface elevation, where an obvious boundary between the cavity and the surface water film can be noticed. The water film later shrinks into a long water column, possibly associated with the surface tension. The bouncing velocity is just $v_{2}=6.22 \mathrm{~m} / \mathrm{s}$, much smaller than the initial impact velocity
Figure 3 Images of cavity evolution for case 5 with $v_{1}=57.88 \mathrm{~m} / \mathrm{s}$ and $\theta_{1}=5.89^{\circ}$

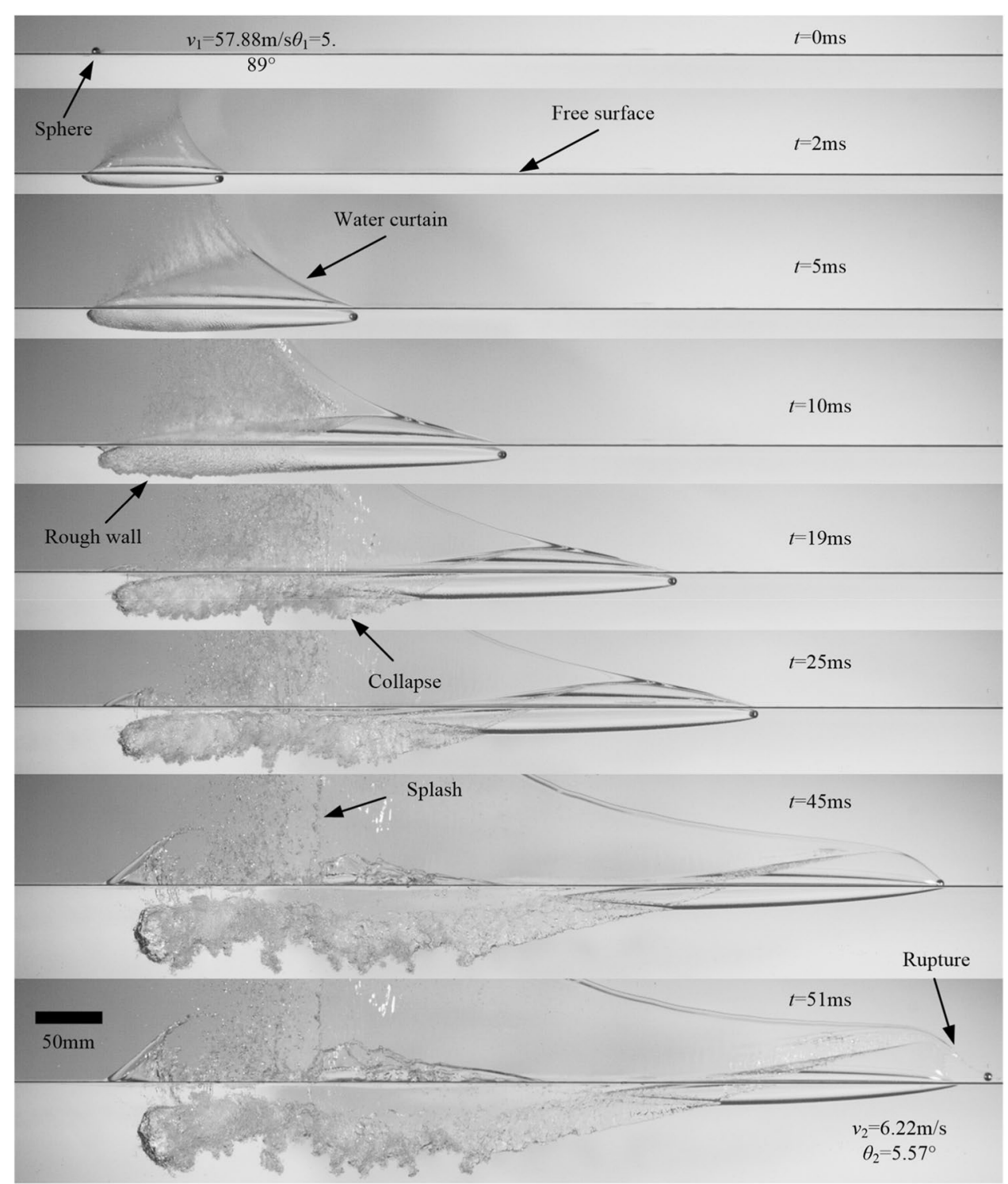


of $v_{1}=57.88 \mathrm{~m} / \mathrm{s}$. More than $98.8 \%$ of the sphere's kinetic energy is lost during the impact process, which unboundedly transfers to the energy of the water film and cavity.

To clearly analyze the phenomenon and mechanism of a sphere bouncing on a water surface, two experimental cases with different initial impact angles were selected. Figure 4 shows the image sequence of cavity evolution for the sphere in case 6 . The initial impact velocity for case 6 $\left(v_{1}=57.68 \mathrm{~m} / \mathrm{s}\right)$ is almost the same as that of case 5 , while the initial impact angle $\left(\theta_{1}=6.01^{\circ}\right)$ is slightly larger. The early-stage flow physics for case 5 and case 6 is quite similar, including the cavity and evolution of water film. Nevertheless, obvious differences are noticed in the flow physics and movement of the sphere for the two cases. In case 6, the sphere eventually enters the water after sliding a long distance near the free surface, as shown in Figure 4. The main reason for the difference is that the lift force of the water on the sphere is less than the gravitational and inertial forces associated with the larger initial impact angle for case 6 . Eventually, the kinetic energy of the sphere gradually dissipates under the free surface and the sphere continues to move underwater.

Figure 5 shows a selected image sequence of the sphere in case 7 hitting the water with a larger initial impact angle of $\theta_{1}=10.23^{\circ}$. The initial impact velocity was $v_{1}=56.78 \mathrm{~m} / \mathrm{s}$, close to those of other cases. It can be seen that the sphere enters directly into the water. A perfect and smooth cavity is formed, which expands and is elongated as the sphere descends in the water. The contraction of the tail of the cavity is obvious under the free surface in Figure 5. Moreover, the water curtain above the free surface has a clear tendency to expand outward, accompanied by the forward movement of the sphere. At the later stage, the cavity wall becomes rough when it collapses because of the effects of hydrostatic pressure and surface tension.

\subsection{Mechanism of a Sphere Bouncing on the Water}

The typical images for four cases are selected to analyze the mechanism of a sphere bouncing on the water. As shown in Figure 6, the cavity length between the impact point and bouncing point for the sphere in the four cases is $90.4,114.8$, 134.7 , and $172.6 \mathrm{~mm}$, respectively. The maximum depth of the cavity in the four bouncing spheres is $4.2,9.1,10.4$, and $14 \mathrm{~mm}$, respectively. The percentage of kinetic energy loss $\eta$ can be estimated from $\eta=\left(v_{1}^{2}-v_{2}^{2}\right) / v_{1}^{2}$, where $v_{1}$ is the initial impact velocity, and $v_{2}$ is the bouncing velocity. This energy loss is used to maintain the development of the cavity. The values of bouncing velocity and $\eta$ for the four cases are shown in Figure 6. It can be seen that, at the same $v_{1}$, the bouncing velocity decreases as the initial angle of incidence increases, which leads to an increase in the percentage of kinetic energy loss. Furthermore, the maximum cavity length and depth increase because of the increase in $\theta_{1}$.

Figure 7 sketches the evolution of the cavity profile and the brief force analysis for case 4 , including the whole
Figure 4 Cavity evolution and movement of a sphere with $v_{1}=57.68 \mathrm{~m} / \mathrm{s}$ and $\theta_{1}=6.01^{\circ}$ for case 6

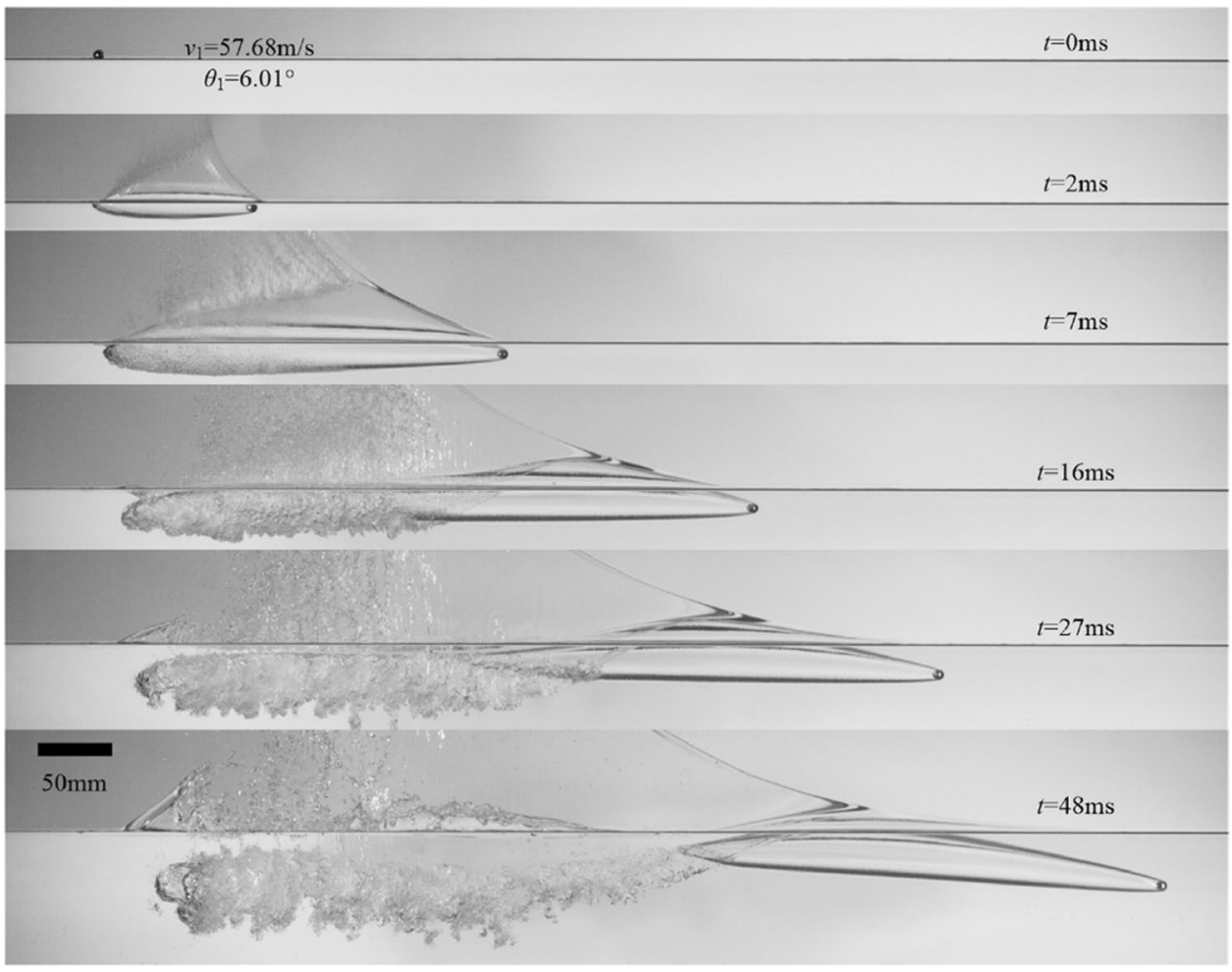


Figure 5 Evolution of the cavity caused by the sphere with $v_{1}=56.78 \mathrm{~m} / \mathrm{s}$ and $\theta_{1}=10.23^{\circ}$ for case 7

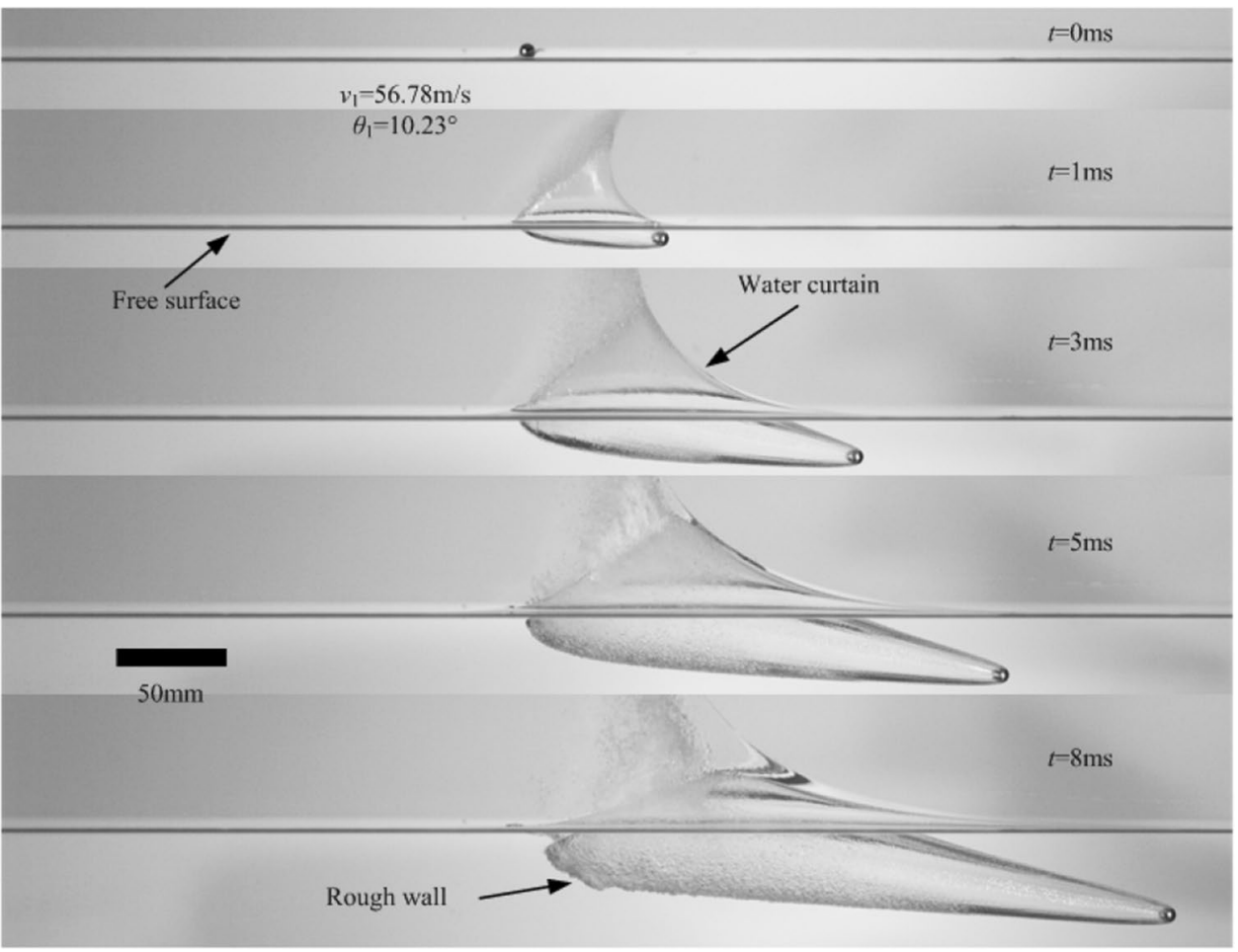

process of the sphere impacting, gliding, and bouncing on the water surface. The blue dotted lines indicate the profile of the splash, and the red lines are the contact area between the sphere and water. Because of the interaction between the sphere and water in the contact area, the sphere is subjected to a lift force, as shown in Figure 7. During the process of the impact, the external forces acting on the sphere are the weight $m g$, the buoyancy force $F_{b}$, the steady-state drag force $F_{d}$, the capillary force $F_{c}$ due to surface tension, and the hydrodynamic force $F_{h}$, also called the resistive force. On the basis of Newton's second law, the equation of motion of a sphere bouncing on the water can be written as follows:
Figure 6 Typical images for cases 1-4 with different initial impact angles

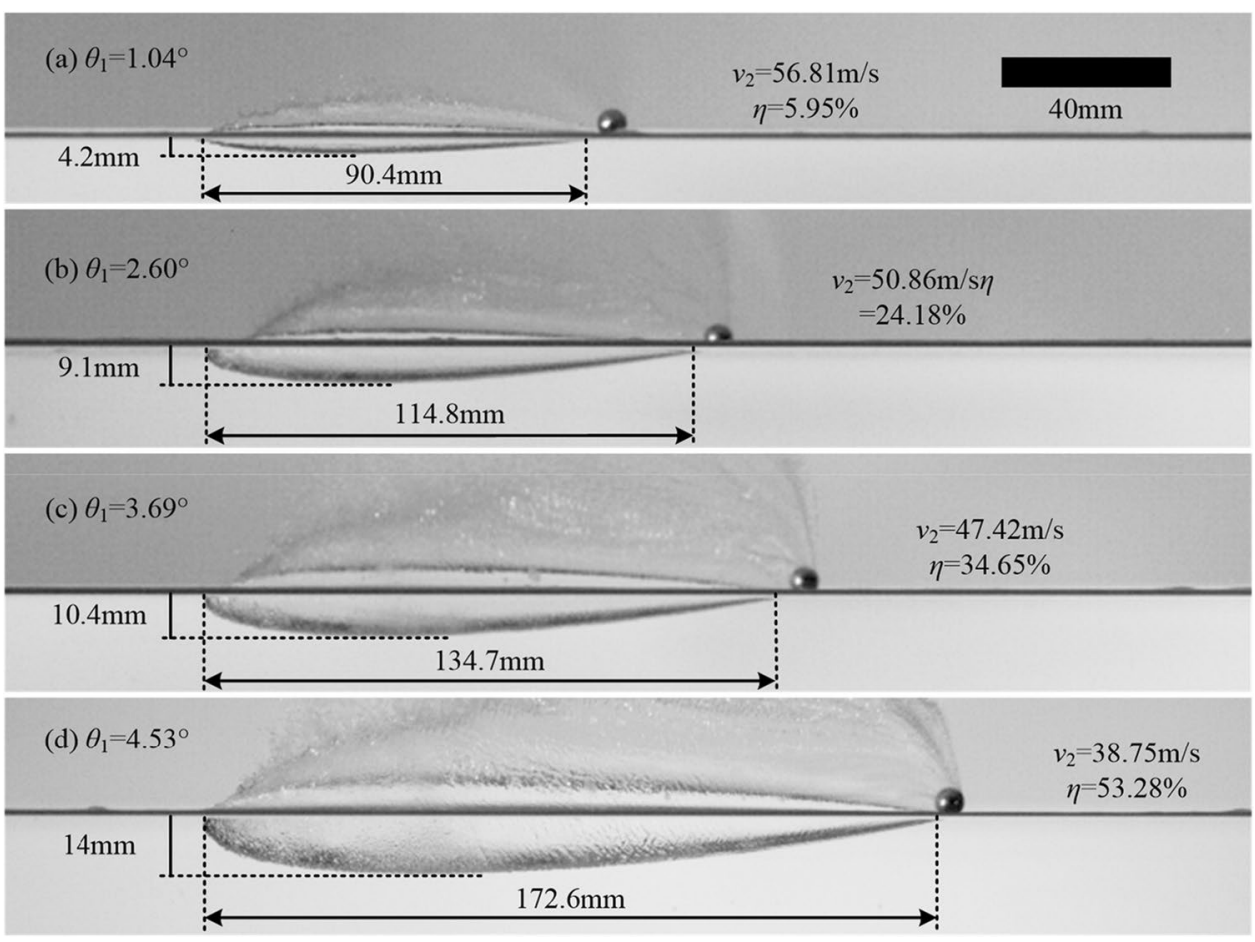




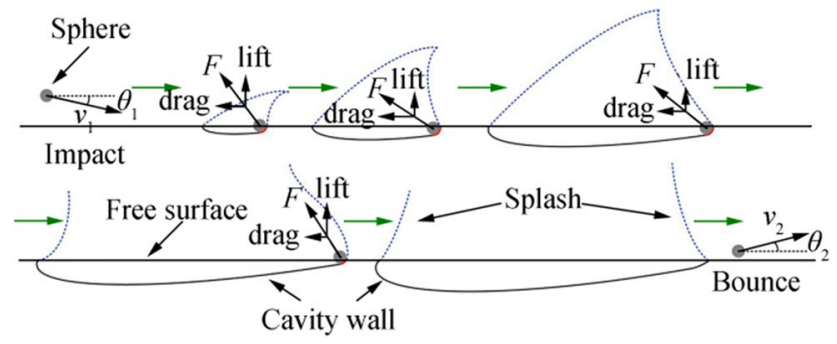

Figure 7 Sketches for cavity configurations and force characteristics analysis of a sphere

$m a=m g-F_{h}-F_{b}-F_{c}-F_{d}$

In the process of the sphere bouncing on the water, the hydrodynamic force $F_{h}$ is the dominant force, and the direction and magnitude of $F_{h}$ change with the direction of movement. The entire force on the sphere can be decomposed into two components: horizontal drag and vertical lift. The drag reduces the velocity, and the lift eventually changes the direction of the sphere. Because of the external forces, the sphere bounces on the free surface.

\subsection{Force Characteristics Analysis of a Sphere Bouncing on the Water}

In this section, the kinematics and dynamic characteristics of a sphere bouncing on a water surface are analyzed quantitatively. Figure 8 presents the displacement, velocity, and acceleration curves associated with cases 5 and 6 in the horizontal and vertical directions. The analytical method is similar to that described by Epps et al. (2010) and Wei and $\mathrm{Hu}(2014,2015)$. The line $(x, 0)$ indicates the static-free surface, and the point $(0,0)$ is the impact point of the sphere on the water. The initial $\theta_{1}$ and $v_{1}$ for the two cases are quite similar, while the impact phenomena are significantly different.

Displacement curves in cases 5 and 6 show that the sphere trajectories are the same at the initial stage of impact (referring to Figure 8(a)). As the sphere moves forward, its trajectory gradually is deflected upward and eventually sends it rushing out of the free surface in case 5 . The obvious difference is that the sphere's trajectory in case 6 shifts downward and is gradually moved away from the static-free surface. The velocity variations of spheres along the $x$ - and $y$-directions are shown in Figure 8(b) and (c). It is found that the tendencies for the two cases are similar along the $x$-direction (Figure 8(b)), while the tendencies along the $y$-direction gradually differ over time, as shown in Figure 8(c). It can be seen that the vertical velocity of the sphere in case 5 changes from negative to positive at $t=11 \mathrm{~ms}$, which indicates that the sphere reaches the deepest point in the cavity and begins to move upward at this time. In contrast, the vertical velocity of the sphere in case 6 almost levels off after a rapid decrease in the early stage of impact.
Figure 8 Variation of parameters for cases 5 and 6

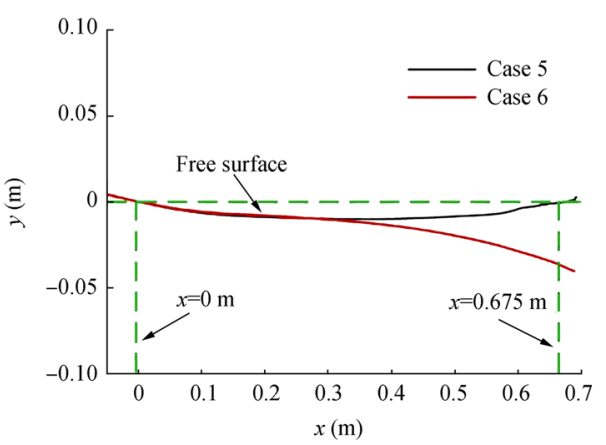

(a) Displacement

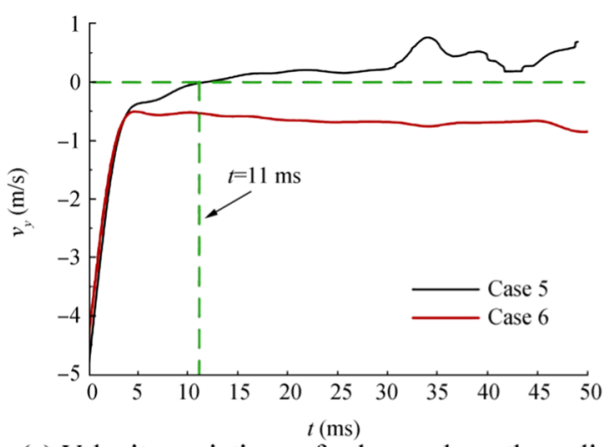

(c) Velocity variations of spheres along the $y$-direction

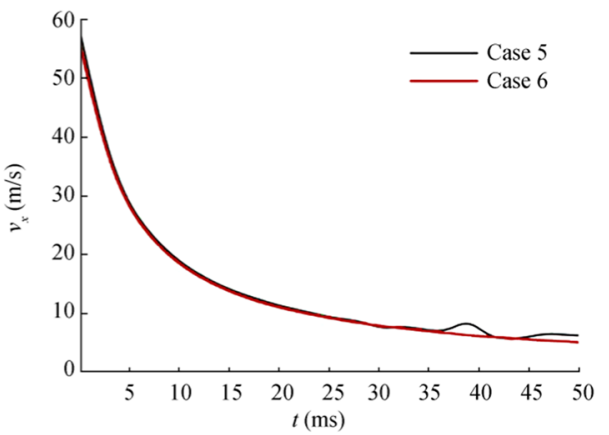

(b) Velocity variations of spheres along the $x$-direction

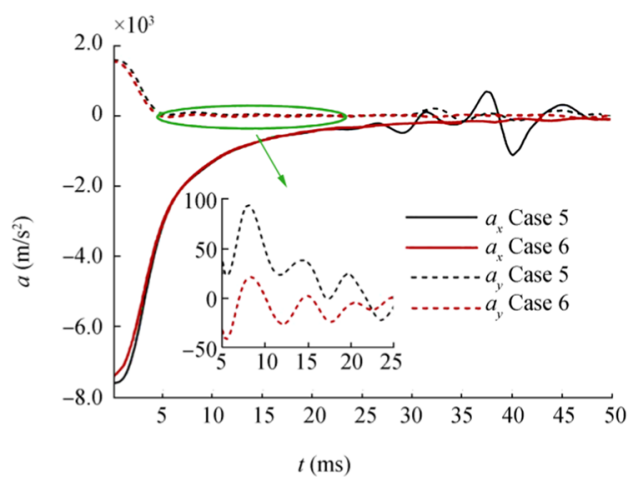

(d) Acceleration 
The acceleration curves in the $x$ - and $y$-directions in Figure 8(d) almost overlap in the early stage. Nevertheless, slight differences are noticed along the $y$-direction after impact, as shown in detail in Figure 8(d). The acceleration in case 5 is positive, which causes the sphere to ascend gradually until rushing out of the water. In contrast, the acceleration in case 6 is negative, which leads the sphere to move downward throughout the process of impacting the water.

\subsection{Statistics of a Successful Bounce}

To reveal the bouncing law of the sphere on a free water surface, numerous experiments have been conducted. A schematic diagram of the statistical distribution of $\theta_{1}$ and $v_{1}$ during the experiments is shown in Figure 9. The black hollow squares stand for the cases in which the sphere bounces on the water surface, and the red solid triangles are the cases without bouncing. A conspicuous boundary is found to differentiate the phenomena of bouncing and non-bouncing. The boundary line is shown with a blue dotted line in Figure 9 and can be fitted as follows:

$v_{1}=17.5 \theta_{1}-45.5$

Definitely, the flow physics of bouncing or no bouncing for a sphere impacting on the free water surface within this velocity range (i.e., $<60 \mathrm{~m} / \mathrm{s}$ ) can be predicted by this boundary line. Figure 9 also indicates that the maximum bounce angle is lower than $6.5^{\circ}$, which is consistent with the law of $\theta_{c}=18 / \sqrt{\sigma}$ given by Johnson and Reid (1975), where $\theta_{c}$ the critical angle and $\sigma$ is the density ratio of the sphere to water. Because of the interference of uncertain

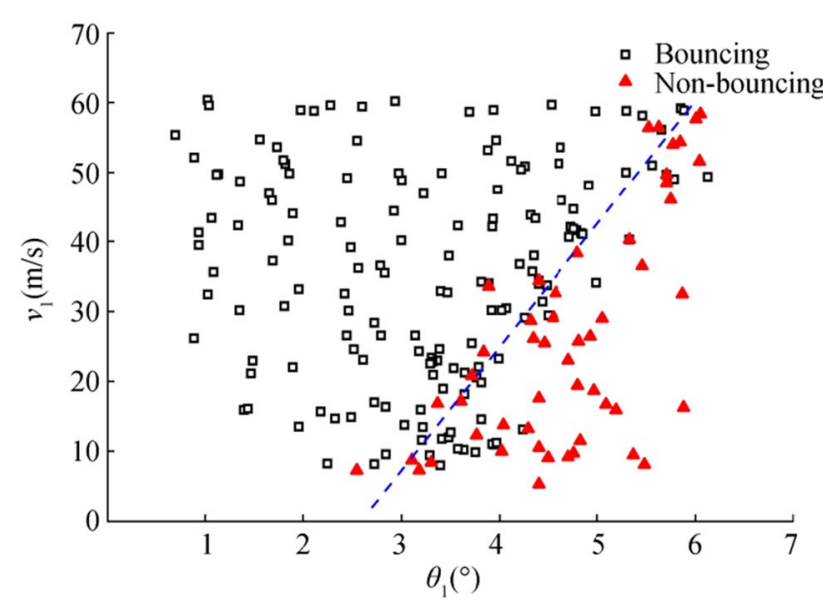

Figure 9 Distribution of the initial impact angle $\left(\theta_{1}\right)$ and initial impact velocity $\left(v_{1}\right)$ factors, some data points are located on the opposite side of the boundary line, which is not in accordance with the law.

For the process of a sphere bouncing on the water, another significant characterization parameter is the bouncing angle $\theta_{2}$. The distribution of $\theta_{2}$ and $\theta_{1}$ is shown in Figure 10. Experimental data points of the bouncing cases can be distinguished through the duration $t$, which is the period from the sphere impacting on the water to leaving the static-free surface. These black solid squares replace the cases in which the duration $t$ is smaller than $10 \mathrm{~ms}$, and the red hollow triangles replace the cases with $t$ larger than $10 \mathrm{~ms}$. Note that the blue dotted line corresponds to $\theta_{1}=\theta_{2}$. It is clearly seen that the statistical data points are mainly distributed near the blue dotted line, and the distribution is more concentrated for the cases of $t \leq 10 \mathrm{~ms}$. For cases with the spheres gliding on the water for a longer time ( $t>10 \mathrm{~ms}$ ), the bouncing angle $\theta_{2}$ tend to be smaller than the initial impact angle $\theta_{1}$.

As mentioned above, the energy is transmitted from the sphere to water during the bouncing process. Figure 11 shows the statistical data points for the variation in kinetic energy loss percentage $\eta$ with bouncing angle. The loss of kinetic energy is more obvious at the moment for the cases with larger initial impact angles. Furthermore, the percentage of kinetic energy loss is below $50 \%$ when the impact angle is smaller than $3^{\circ}$. The energy attenuation rate corresponding to different angles is between two curves for all the cases, as shown in Figure 11, and the two curves can be fitted using the following equations:

$\eta=8.67 \theta_{1}^{2}-3.33 \theta_{1}$

$\eta=2.13 \theta_{1}^{2}-0.5 \theta_{1}$

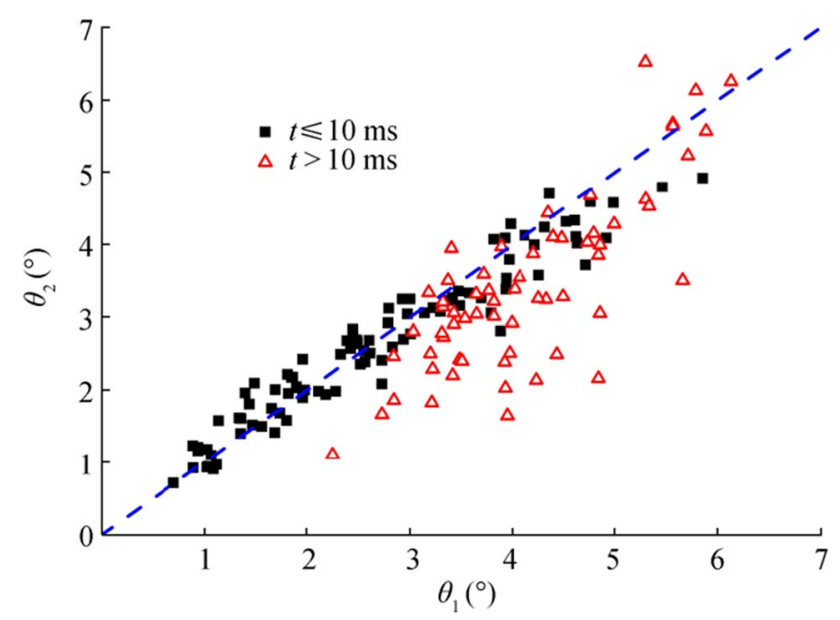

Figure 10 Distribution of initial impact angle $\left(\theta_{1}\right)$ and bouncing angle distributions $\left(\theta_{2}\right)$ 


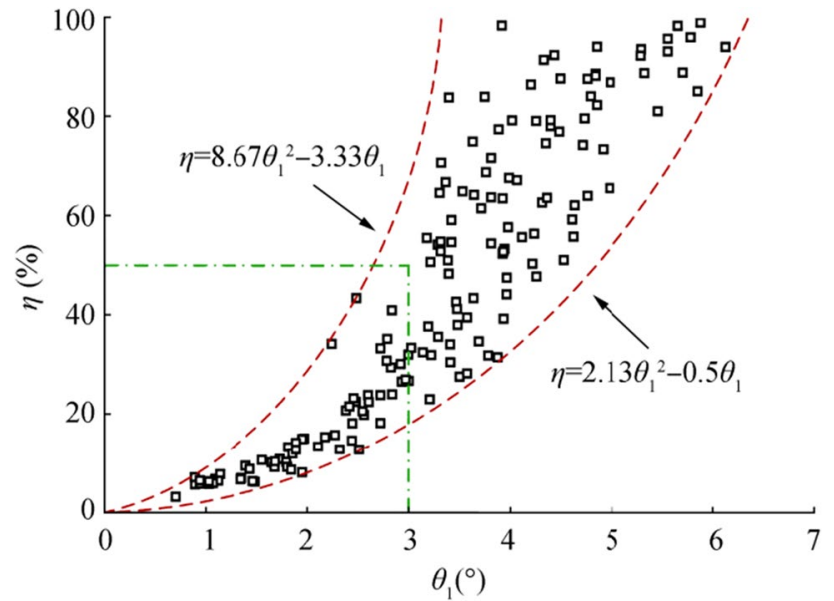

Figure 11 Variation of the percentage of kinetic energy loss $(\eta)$ with bouncing angle $\left(\theta_{1}\right)$

\section{Conclusions}

In this work, the cavity configurations and kinematic characteristics of a sphere bouncing on water are experimentally investigated. Through continuous improvement of experimental methods, high-resolution images of the cavity evolution of a sphere bouncing on the water are obtained, and they show the evolution process of cavitation more intuitively and facilitate the analysis of complex flow phenomena during the process. The motion and force characteristics of the sphere in typical cases are extracted and analyzed by an advanced image processing technique. More than 200 cases of a sphere impacting on a water surface have been considered, and the effect of initial impact velocity and initial impact angle on the phenomena of sphere bouncing has been investigated. The results indicate that a critical curve of $v_{1}=17.5 \theta_{1}-45.5$ shows the relationship between the initial velocity and angle and determines whether the sphere bounces on the water surface. In addition, for the impacting cases with the same initial velocity, the energy attenuation of the sphere is closely associated with the initial impact angle during bouncing, and more than half of the kinetic energy is lost for an impact angle of $\theta_{1}>3^{\circ}$.

Funding Supported by the Fundamental Research Funds for the Central Universities (30918012201) and the Fund of the State Key Laboratory (6142604190302).

Open Access This article is licensed under a Creative Commons Attribution 4.0 International License, which permits use, sharing, adaptation, distribution and reproduction in any medium or format, as long as you give appropriate credit to the original author(s) and the source, provide a link to the Creative Commons licence, and indicate if changes were made. The images or other third party material in this article are included in the article's Creative Commons licence, unless indicated otherwise in a credit line to the material. If material is not included in the article's Creative Commons licence and your intended use is not permitted by statutory regulation or exceeds the permitted use, you will need to obtain permission directly from the copyright holder. To view a copy of this licence, visit http://creativecommons.org/licenses/by/4.0/.

\section{References}

Belden J, Hurd RC, Jandron MA, Bower AF, Truscott TT (2016) Elastic spheres can walk on the water. Nat Commun 7:10551

Bocquet L (2003) The physics of stone skipping. Am J Phys 71:150-155

Clanet C, Hersen F, Bocquet L (2004) Secrets of successful stoneskipping. Nature 427(6969): 29

Epps BP, Truscott TT, Techet AH (2010) Evaluating derivatives of experimental data using smoothing splines. Mathematical Methods in Engineering International Symposium, 29-38

Faltinsen OM (2000) Hydroelastic Slamming. J Mar Sci Tech-Japan 5:49-65

Glasheen JW, Mcmahon TA (1996) A hydrodynamical model of locomotion in the basilisk lizard. Nature 380:340-342

Grumstrup T, Keller JB, Belmonte A (2007) Cavity ripples observed during the impact of solid objects into liquids. Phys Rev Lett 99:114502

Hewitt IJ, Balmforth NJ, Mcelwaine JN (2011) Continual skipping on the water. J Fluid Mech 669:328-353

Hicks PD, Smith FT (2011) Skimming impacts and rebounds on shallow liquid layers. Proceedings: Mathematical. Phys Eng Sci 467:653-674

Hurd RC, Belden J, Bower AF, Holekamp S, Jandron MA, Truscott TT (2019) Water walking as a new mode of free surface skipping. Sci Rep-UK 9:6042

Johnson W (1998) Ricochet of non-spinning projectiles, mainly from water part I: some historical contributions. Int J Impact Eng 21:15-24

Johnson W, Reid SR (1975) Ricochet of spheres off water. J Mech Eng Sci 17:71-81

Korobkin A, Parau EI, Vanden-Broeck JM (2011) The mathematical challenges and modelling of hydroelasticity. Philos Trans R Soc A: Math Phys Eng Sci 369:2803-2812

Liu K, Smith FT (2014) Collisions, rebounds and skimming. Philos Trans A Math Phys Eng Sci 372:20130351

Miloh T, Shukron Y (1991) Ricochet off water of spherical projectiles. J Ship Res 35:91-100

Nishida M, Okumura M, Tanaka K (2010) Effects of density ratio and diameter ratio on critical incident angles of projectiles impacting granular media. Granul Matter 12:337-344

Rosellini L, Hersen F, Clanet C, Bocquet L (2005) Skipping stones. J Fluid Mech 543:137

Shlien DJ (1994) Unexpected ricochet of spheres off water. Exp Fluids 17:267-271

Truscott TT, Epps BP, Belden J (2014) Water entry of projectiles. Annu Rev Fluid Mech 46:355-378

Von Karman T (1929) The impact on seaplane floats during landing. NACA Technical Note, 321

Wei ZY, Hu CH (2014) An experimental study on the water entry of horizontal cylinders. J Mar Sci Tech-Japan 19:338-350

Wei ZY, Hu CH (2015) Experimental study on the water entry of circular cylinders with inclined angles. J Mar Sci Tech-Japan 20:722-738

Yun HL, Lyu XJ, Wei ZY (2020) Experimental study on vertical water entry of two tandem spheres. Ocean Eng 201:107143 